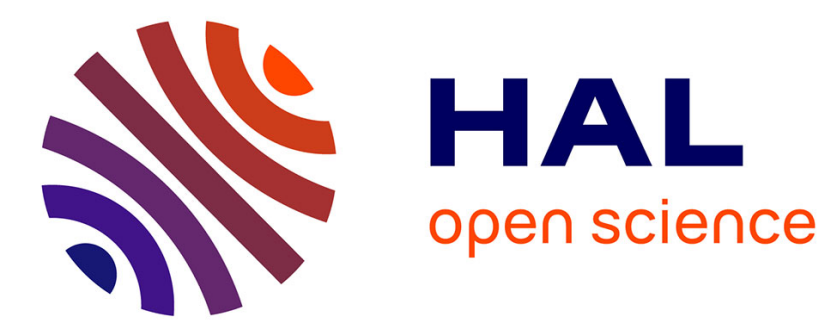

\title{
Dérivation numérique d'une collection de points
}

\author{
G. Bastide, G. Sagnes
}

\section{To cite this version:}

G. Bastide, G. Sagnes. Dérivation numérique d'une collection de points. Revue de Physique Appliquée, 1982, 17 (11), pp.769-774. 10.1051/rphysap:019820017011076900 . jpa-00245055

\section{HAL Id: jpa-00245055 https://hal.science/jpa-00245055}

Submitted on 1 Jan 1982

HAL is a multi-disciplinary open access archive for the deposit and dissemination of scientific research documents, whether they are published or not. The documents may come from teaching and research institutions in France or abroad, or from public or private research centers.
L'archive ouverte pluridisciplinaire HAL, est destinée au dépôt et à la diffusion de documents scientifiques de niveau recherche, publiés ou non, émanant des établissements d'enseignement et de recherche français ou étrangers, des laboratoires publics ou privés. 
Classification

Physics Abstracts

$72.80 \mathrm{Ey}-71.50+\mathrm{t}$

\title{
Dérivation numérique d'une collection de points
}

\author{
G. Bastide et G. Sagnes \\ Université des Sciences et Techniques du Languedoc, Centre d'Etudes d'Electronique des Solides, \\ associé au C.N.R.S., Place E. Bataillon, 34060 Montpellier Cedex, France
}

(Reçu le 9 mars 1982, révisé le 7 juin 1982, accepté le 21 juillet 1982)

\begin{abstract}
Résumé. - Nous présentons une technique de dérivation numérique qui procure une réjection du bruit de $18 \mathrm{~dB} /$ octave. Après détermination des coefficients de la fonction de transfert en $Z$ du dérivateur, le calcul de la dérivée se réduit à une combinaison linéaire des 5 éléments d'un tableau. Le traitement peut être effectué en temps réel.
\end{abstract}

\begin{abstract}
We report a numerical method to calculate the sampled derived function which gives a noise rejection of $18 \mathrm{~dB} /$ octave. After a preliminary calculation of the coefficients of the $Z$ transfer function of the filter the derivative is obtained by a linear combination of the 5 elements of an array. The treatment may be performed in real time operation.
\end{abstract}

1. Introduction. - La plupart des appareils de mesure modernes sont numériques et on estime qu'à la fin 1982 deux à trois mille d'entre eux seront équipés de l'interface IEEE 488. Cette interface leur procure la possibilité de converser très facilement avec toutes sortes de calculateurs qui sont eux-mêmes bon marché, très performants et programmables en langage évolué. Ces calculateurs peuvent d'ailleurs participer activement au contrôle du processus expérimental en modifiant certains paramètres de l'expérience et en interrogeant les appareils de mesure. Ils peuvent effectuer en temps réel des calculs élémentaires sur les données : conversions d'échelles, linéarisation de capteurs, tracés de courbes, etc. La présence des calculateurs procure un certain nombre d'avantages supplémentaires. Ils sont capables, par exemple, d'acquérir des transitoires très rapides ou au contraire extrêmement lents et de constituer ainsi des fichiers de données expérimentales qu'on peut réutiliser autant de fois que nécessaire pour en tirer le maximum d'information possible. Beaucoup d'expériences sont donc, à l'heure actuelle, pilotées par calculateur et l'information qu'elles collectent ne peut être traitée que par des techniques numériques. La plupart de ces traitements, pourtant complexes, que l'on peut appliquer à ces données ne posent pas de problèmes particuliers. C'est le cas des processus qui font intervenir une intégration ; filtrage passe-bas, corrélation, convolution, transformation de Fourier, etc. Par contre, les opérations de filtrage passe haut, même du $1^{\mathrm{er}}$ ordre comme la déri- vation, ne peuvent pas être appliquées directement à cause de la dégradation importante du rapport signal sur bruit qu'elles entraînent. Le but de cet article est précisément de donner une technique de dérivation numérique qui atténue considérablement cet inconvénient.

Les performances de cette méthode sont démontrées par application à une courbe d'équation connue, puis elle est appliquée à la dérivation d'une courbe de capacité stimulée thermiquement (TSCAP). Le déplacement du point d'inflexion de la TSCAP avec le taux de chauffage donne l'énergie d'activation du niveau profond concerné.

2. Exposé de la méthode. - 2.1 FonCtiON DE TRANSFERT DU FILTRE NUMÉRIQUE. - La figure $1 a$ est l'exemple typique d'une courbe qu'il est nécessaire de dériver si l'on veut préciser la position du point d'inflexion. Ni un changement d'échelle ni un décalage du zéro ne modifieraient suffisamment sa forme pour qu'on puisse se dispenser de cette opération. Cette courbe a pour équation :

$$
y=1+x+2 \sin \frac{\pi(x-5)}{10}+0,002 x^{3}+\text { bruit }
$$

Le bruit d'amplitude 0,02 est simulé par le générateur de nombres pseudo-aléatoires du calculateur. Le tracé est effectué par pas de $\Delta x=0,05$ et correspond donc à une collection de 201 points expérimentaux $y_{i}$ échantillonnés à la même période $T=0,05$. 

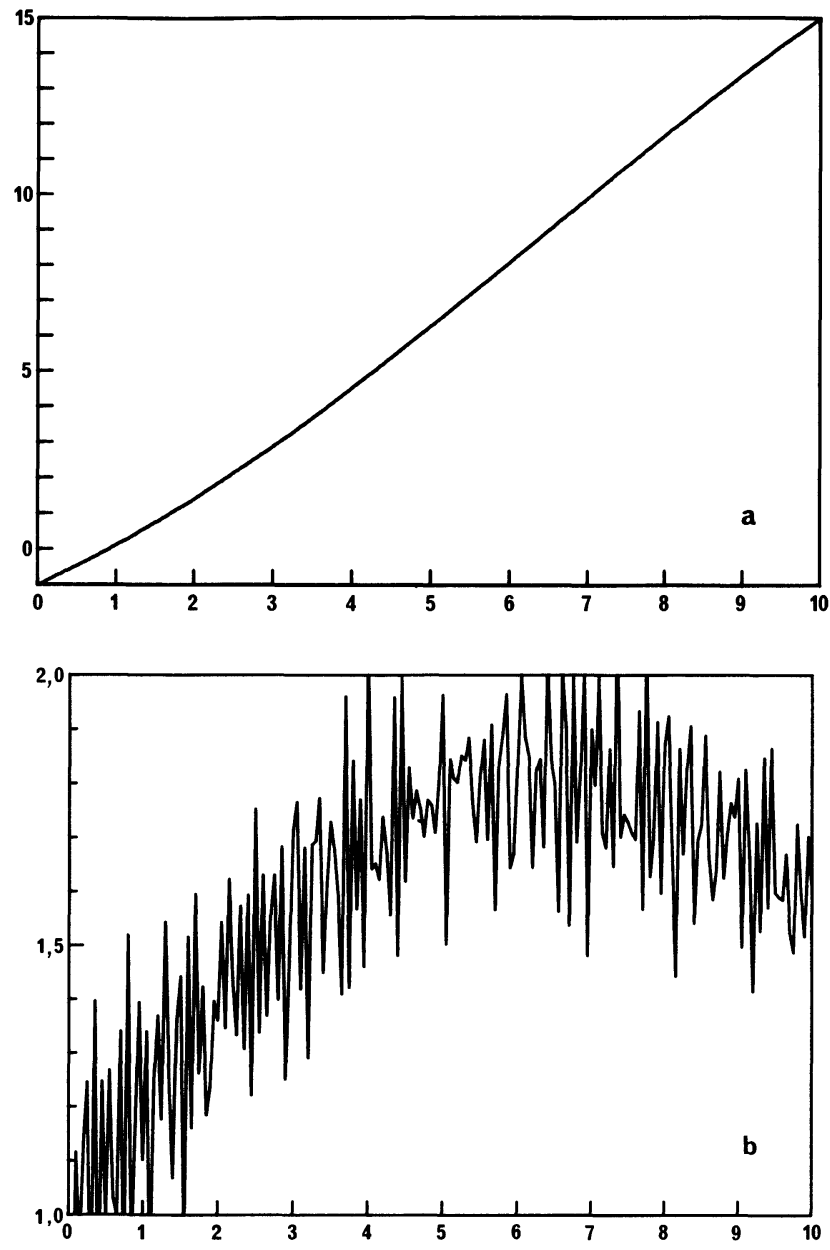

Fig. 1. - a) Courbe calculée d'équation

$$
y=1+x+\sin \frac{\pi(x-5)}{10}+0,002 x^{3}
$$

sur laquelle il faut préciser la position du point d'inflexion. b) Dérivée numérique de la courbe $1 a$ obtenue par l'équation $y^{\prime}=\Delta y / T$

[a) Calculated curve of equation

$$
y=1+x+\sin \frac{\pi(x-5)}{10}+0.002 x^{3}
$$

on which one has to localize the position of the inflexion point. b) Numerical derivative of the curve of figure $1 a$ obtained by using $y^{\prime}=\Delta y / T$.]

La façon la plus évidente pour obtenir la dérivée est de calculer :

$$
\frac{y_{i+1}-y_{i}}{T}=f(i T) \text {. }
$$

Le résultat catastrophique de cette opération est montré sur la figure $1 b$. La dégradation du rapport $\mathrm{S} / \mathrm{B}$ qu'on observe n'est pas particulière à la dérivation numérique. Elle se produirait de façon identique dans un dérivateur électronique traitant directement le signal analogique. Le remède est d'ailleurs classique en analogique. Il consiste à remplacer le dérivateur vrai, de fonction de transfert symbolique $H(s)=s$, soit $H(j \omega)=j \omega$, par un dispositif qui dérive les basses fréquences et qui intègre les hautes fréquences, c'est-à-dire principalement le bruit. Le montage le plus souvent utilisé est le dérivateur en pont de Wien dont la fonction de transfert est du type :

$$
H_{\omega}(s)=\frac{s \omega_{0}^{2}}{\omega_{0}^{2}+2 \omega_{0} s+s^{2}} .
$$

C'est un filtre passe bande de fréquence centrale $f_{0}=\omega_{0} / 2 \pi$. Avec un tel dispositif, seuls les signaux de fréquence assez nettement inférieure à $f_{0}$ (i.e. $\left.f<f_{0} / 2\right)$ sont dérivés correctement, alors que les signaux à variation rapide $\left(f>2 f_{0}\right)$ sont atténués (intégrés $\left.H_{\omega}(s) \cong 1 / s\right)$ à raison de $6 \mathrm{~dB} /$ octave. La même approximation pourrait être adoptée pour la dérivation numérique, mais on peut, dans le but d'appliquer cette technique, prévoir une approximation plus performante pour la réjection du bruit. On peut par exemple, utiliser une fonction de transfert à $+6 \mathrm{~dB} /$ octave en $\mathrm{BF}$

$$
H(s)=s
$$

qui assure la dérivation et à $-18 \mathrm{~dB} /$ octave en $\mathrm{HF}$

$$
H(s)=1 / s^{3}
$$

qui filtre le bruit, c'est-à-dire :

$$
H(s)=\frac{s}{P\left(s^{4}\right)}
$$

où $P\left(s^{4}\right)$ est un polynôme de degré 4 en $s$.

Il est commode de prendre pour $P$ le polynôme de Butterworth d'ordre 4 qui conduit à un filtre stable de fonction de transfert parfaitement linéaire $(H(s)=s)$ dans un domaine étendu de fréquence.

La fonction de transfert adoptée en définitive pour une dérivation analogique est du type :

$$
H(s)=\frac{s}{s^{4}+(A+B) s^{3}+(2+A B) s^{2}+(A+B) s+1}
$$

avec

$$
\begin{aligned}
& A=-2 \cos (5 \pi / 8) \\
& B=-2 \cos (7 \pi / 8) .
\end{aligned}
$$

Dans un traitement numérique on opère non pas sur une variable continue, mais sur une suite discrète de valeurs mesurées (échantillonnées) avec une période $T$. La caractéristique du filtre n'est pas, pour ces signaux discontinus, décrite par la fonction $H(s)$ mais par la fonction de transfert en $Z, H(Z)=s(Z) / y(Z)$, rapport entre les transformées $Z$ des séquences de sortie $s(n T)$ et d'entrée $y(n T)(n=0,1,2 \ldots)$. Pour cette application, on peut obtenir $H(Z)$ à partir de $H(s)$ par la transformation conforme de Kaiser et Golden [1] et Rader et Gold [2] :

$$
s=\frac{1}{K} \frac{Z-1}{Z+1} \quad \text { avec } \quad K=\operatorname{tg} \frac{\omega_{0} T}{2}
$$

où $f_{0}=\omega_{0} / 2 \pi$ est la fréquence centrale du filtre. 
On obtient alors

$$
\frac{s(Z)}{y(Z)}=H(Z)=\frac{2}{T} \frac{D_{0} / B_{5}\left(1+2 / Z-2 / Z^{3}-1 / Z^{4}\right)}{1+B_{4} / Z+B_{3} / Z^{2}+B_{2} / Z^{3}+B_{1} / Z^{4}}=\frac{N(1 / Z)}{D(1 / Z)}
$$

avec :

$$
\begin{array}{ll}
D_{0}=K^{4} & B_{5}=1+D_{0}+D_{1}+D_{2}+D_{3} \\
D_{1}=K^{3}(A+B) & B_{4}=\left(-4-2 D_{3}+2 D_{1}+4 D_{0}\right) / B_{5} \\
D_{2}=K^{2}(2+A B) & B_{3}=\left(6-2 D_{2}+6 D_{0}\right) / B_{5} \\
D_{3}=K(A+B) & B_{2}=\left(-4+2 D_{3}-2 D_{1}+4 D_{0}\right) / B_{5} \\
& B_{1}=\left(1-D_{3}+D_{2}-D_{1}+D_{0}\right) / B_{5} .
\end{array}
$$

$X(Z)$ étant la transformée $Z$ d'une séquence $x(n T)$, $n=0,1, \ldots$. On montre facilement que $X(Z) / Z$ a pour original la même séquence retardée d'une période d'échantillonnage. L'équation (1) écrite sous la forme $s(Z) \cdot D(1 / Z)=y(Z) \cdot N(1 / Z)$ permet donc de calculer la valeur de l'échantillon $s(n T)$ par une combinaison linéaire de 5 échantillons d'entrée et 4 échantillons de sortie consécutifs. Il est toutefois plus commode d'écrire $H(Z)$ sous la forme :

soit

$$
H(Z)=\frac{N}{W} \cdot \frac{W}{D}
$$

et

$$
s(Z)=N(1 / Z) \cdot W(1 / Z)
$$

$$
y(Z)=W(1 / Z) \cdot D(1 / Z)
$$

où $W$ est une séquence intermédiaire de 5 échantillons consécutifs : $W_{5}$ à $W_{1}$.

On en déduit l'organisation du filtre numérique schématisée sur la figure 2. Cette figure constitue d'ailleurs pratiquement l'organigramme du calcul de la dérivée.

$W_{5}$ est calculé à partir de l'échantillon d'entrée $y(n T)$ par

$W_{5}=y(n T)-B_{4} W_{4}-B_{3} W_{3}-B_{2} W_{2}-B_{1} W_{1}$ et la valeur de l'échantillon de sortie par

$$
y^{\prime}(n T)=\frac{2}{T} \frac{D_{0}}{B_{5}}\left(-W_{1}-2 W_{2}+2 W_{4}+W_{5}\right) .
$$

2.2 Mise en ceuvre du filtre. - Le calcul de la dérivée proprement dite se réduit aux 4 opérations simples et rapides suivantes :

a) prise de l'échantillon $y(n T)$ sur l'appareil de mesure numérique ;

b) décalage du tableau $W$, i.e.

$$
W_{2} \rightarrow W_{1}, \ldots, W_{5} \rightarrow W_{4}
$$

c) calcul de $W_{5}$ à l'aide de l'équation (2);

d) calcul de $y^{\prime}(n T)$ à l'aide de l'équation (3);

et retour en $a$ ) pour prise de l'échantillon suivant $y(n T+T)$.

Il est donc tout à fait possible, dans la plupart des cas, d'effectuer ce traitement en temps réel, c'est-à-dire au moment même de l'acquisition.

Le tableau $W$ doit naturellement être initialisé à la mise en route (i.e. avant l'acquisition du premier échantillon). En temps réel, la seule possibilité effective est de l'initialiser à 0 et la dérivée n'est correcte qu'après extinction du transitoire de mise en route.

En temps différé, il est possible de réduire la durée (2) de ce transitoire. Il est facile de montrer à partir de

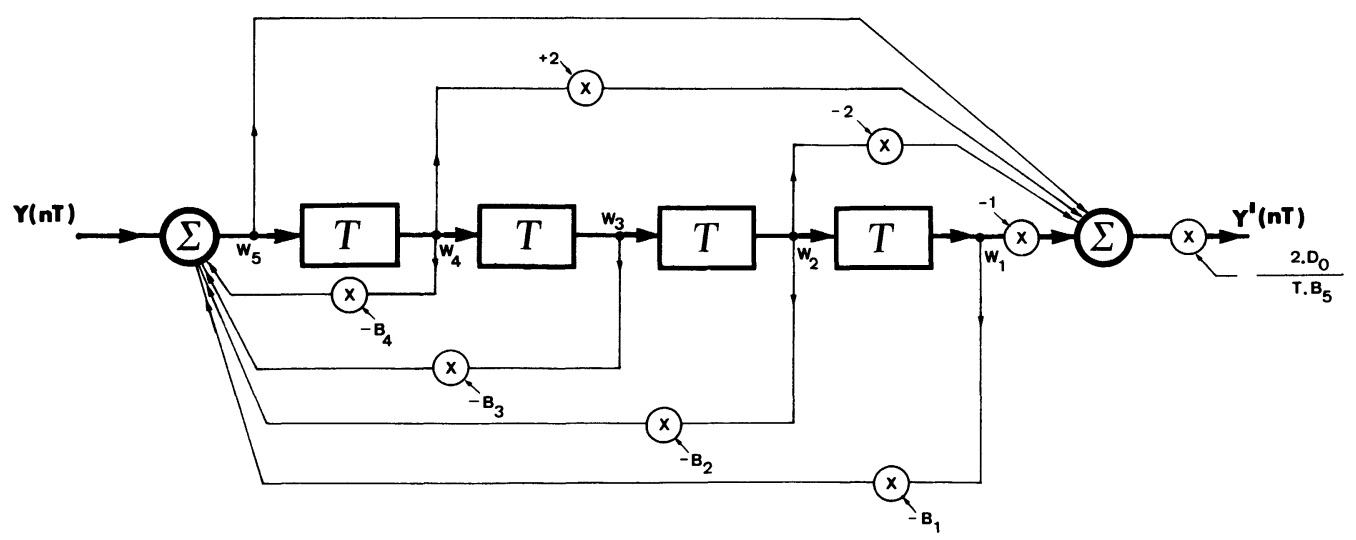

Fig. 2. - Organisation du dérivateur numérique. L'élément symbolisé par $T$ représente un retard d'une période d'échantillonnage.

[Organization of the numerical diffentiator. The element referenced by $T$ represents the delay of one sampling period.] 



Fig. 3. - $-a$ ) Dérivée mathématique de la courbe $1 a . b$ ) Dérivée numérique obtenue pour une fréquence centrale du filtre $\left.f_{0}=1 / 6 T . c\right)$ id. à $3 b$ pour $\left.f_{0}=1 / 30 T . d\right)$ id. à $3 b$ pour $f_{0}=1 / 35 T$ et traitement direct et rétrograde.

[a) Mathematical derivative of curve $1 a . b$ ) Numerical derivative obtained for a central frequency $f_{0}=1 / 6 T$. c) Same as $3 b$ but for $f=1 / 30 T . d$ ) Same as $3 b$ but and with an upward downward treatment and $f_{0}=1 / 35 T$.]

l'équation (3) que lorsque la dérivée $y^{\prime}$ est constante, les $W_{i}$ sont équidistants de :

$$
\alpha=\frac{1}{8} \frac{T}{2} \frac{B_{5}}{D_{0}} y^{\prime} .
$$

On calcule donc, par exemple par une méthode des moindres carrés appliquée à quelques échantillons, une valeur approximative $y_{0}^{\prime}$ de la dérivée initiale, puis $W_{5}(0)$ à partir de l'équation (2)

$$
W_{5}(0)=\frac{y_{0}+\alpha\left(B_{4}+2 B_{3}+3 B_{2}+4 B_{1}\right)}{1+B_{1}+B_{2}+B_{3}+B_{4}}
$$

puis :

$$
W_{4}(0)=W_{5}(0)-\alpha, \quad \ldots W_{1}(0)=W_{2}(0)-\alpha
$$

et on commence le traitement de la séquence $y(n T)$.

Le résultat de ces opérations est montré sur les figures $3 b$ et $3 c$ tracées pour 2 valeurs de la période centrale $T_{0}=1 / f_{0}$ du filtre : $T_{0}=6 T$ et $T_{0}=30 T$. Sur la figure $3 c$ le bruit est pratiquement complètement éliminé, mais le retard introduit par le filtrage passe bas peut être considéré comme excessif (décalage de la position du maximum). En temps différé, on peut à nouveau minimiser cet inconvénient en traitant la collection de points dans le sens normal $S^{+}(n T)$, puis dans le sens rétrograde $S^{-}(n T)$ et en calculant $y^{\prime}(n T)=\frac{1}{2}\left(S^{+}(n T)-S^{-}(n T)\right)$. Le résultat de ce traitement est donné sur la figure $3 d$ pour $T_{0}=35 T$ que l'on peut comparer à la dérivée exacte calculée mathématiquement, figure $3 a$.

2.3 Calculs Préliminaires : CHOIX De $T_{0}$ ET $T$. Ces calculs concernent les coefficients $K, D_{0}$ à $D_{3}$, $B_{1}$ à $B_{5}$. Tous ces coefficients dépendent de $T_{0}$ et $T$. Du choix de $T_{0}$ dépend l'efficacité de la réjection du bruit. Si le signal à traiter est assez " bruyant ", on est condúit à utiliser des grandes valeurs de $T_{0}$ (i.e. $T_{0} \cong 30 T$ ). Cela se traduit par un certain nombre d'inconvénients :

- incapacité à dériver des signaux à variation rapide;

- inexactitude relative de la dérivée (transitoire de mise en route assez long, possible décalage de la position du maximum, etc.). 

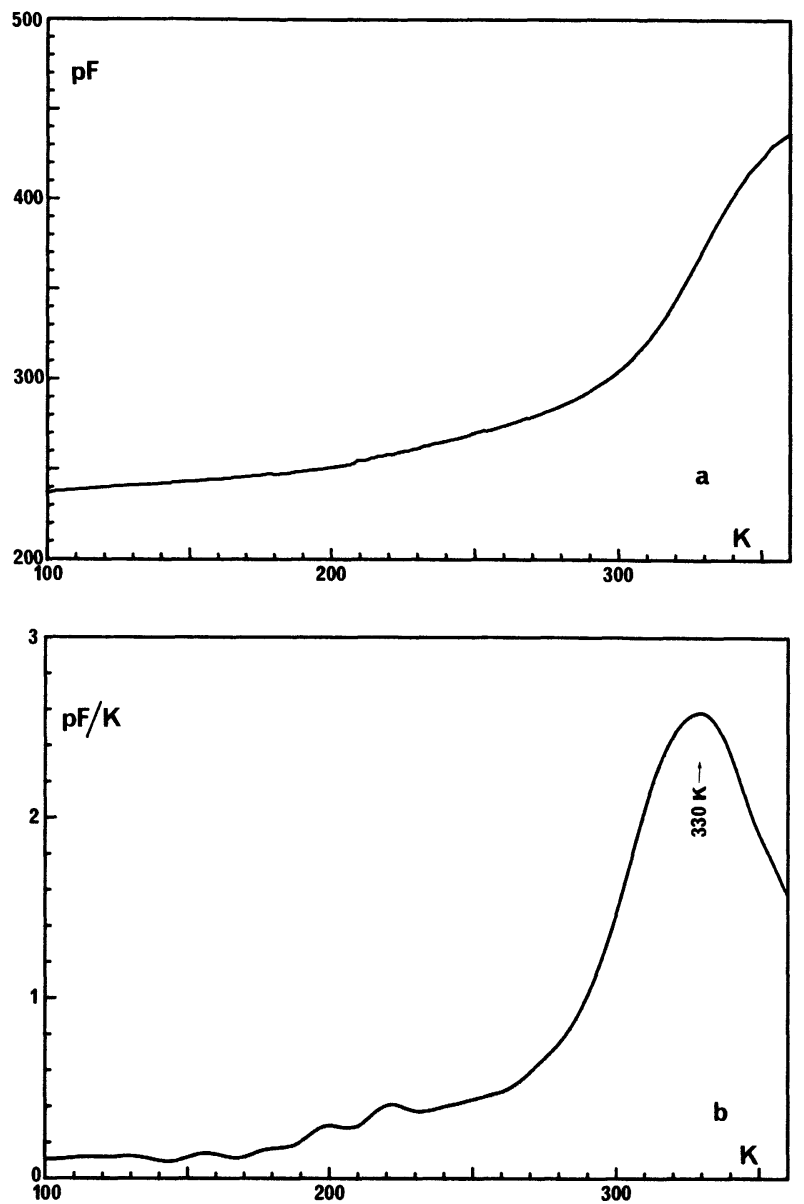

Fig. 4. - a) Courbe de TSCAP d'une jonction or-CdS. Le taux de chauffage est $4 \mathrm{~K} / \mathrm{min}$. b) Dérivée de la courbe $4 a$. La période propre du filtre est $20 \mathrm{~K}$ et le traitement direct et rétrograde.

[a) TSCAP of an Au-CdS Schottky diode. b) Numerical derivative of figure $4 a$. Up-down treatment is used and the central frequency of the filter correspond to $20 \mathrm{~K}$.]

Si au contraire le signal est peu bruyant, on peut utiliser une faible valeur de $T_{0}$, ce qui atténuera les inconvénients ci-dessus. Toutefois $T_{0}$ doit toujours être plus grand de $T$, soit $T_{0}>3$ ou $4 T$. La valeur optimale de $T_{0}$ est le plus souvent déterminée par tâtonnements, d'où l'intérêt d'un traitement en temps différé. Il reste à fixer la période $T$ d'échantillonnage, c'est-à-dire la variation $\Delta t$ que subit le paramètre $t=n T$ au cours d'un cycle complet de l'expérience. Dans certains cas, le calculateur contrôle directement $t$, par exemple pour observer la caractéristique courant/ tension d'un dipôle en commandant le générateur de tension $(t=V)$. Il peut alors, au cours du cycle, faire tous les calculs ou opérations d'entrée/sortie nécessaires même s'ils sont longs ou de durée variable.

Dans d'autres cas, le calculateur ne peut que mesurer le paramètre $t$ ( $t=$ temps, température, etc.). $A$ priori on a intérêt à échantillonner le plus rapidement possible pour procurer le maximum d'information (plus grand nombre de points) à la séquence $y(n T)$. Dans la pratique, il est souvent nécessaire de réduire la fréquence d'échantillonnage :

- en effet, il est impératif que $T$ soit le plus constant possible, c'est-à-dire que $T$ doit être beaucoup plus grand que la résolution de l'appareil qui mesure ou fournit le paramètre $t$.

On peut alors en profiter pour mesurer $y(n T)$ avec un appareil à intégration qui augmente le rapport signal/bruit (un voltmètre qui intègre pendant $20 \mathrm{~ms}$, élimine le bruit à $50 \mathrm{~Hz}$ ). S'il est expérimentalement impossible d'échantillonner à fréquence constante, il faut faire l'acquisition du signal $y(t)$ dans les meilleures

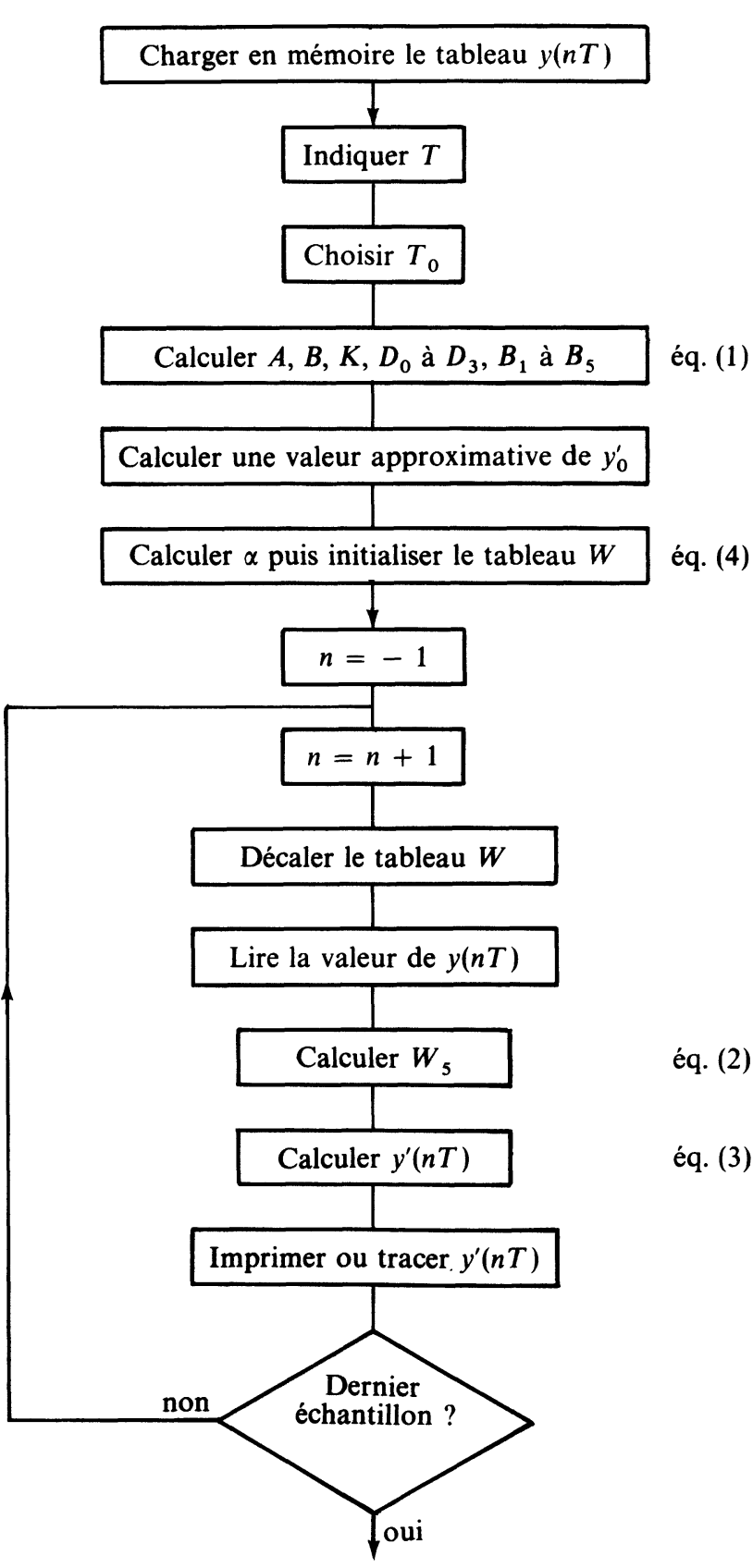

Fig. 5. - Organigramme des opérations à effectuer pour calculer la dérivée en temps différé.

[Flowchart of the calculations required to perform the numerical derivative in delayed time operation.] 
conditions possibles, puis en temps différé, par interpolation, recalculer les échantillons $y(n T)$ avant de les présenter à la dérivation. L'ensemble des opérations à effectuer pour calculer la dérivée en temps différé est rassemblé sur la figure 5 .

2.4 RÉSULTATS. - Les figures $4 a$ et $4 b$ représentent une courbe expérimentale de TSCAP et sa dérivée obtenues sur une diode Schottky or-CdS. Le déplacement du point d'inflexion avec le taux de chauffage donne une énergie d'activation $E=1 \mathrm{eV}$ pour le niveau profond correspondant. D'autres expériences de DLTS et DLOS [3] indiquent qu'il s'agit d'un niveau très fortement relaxé, en très forte interaction avec le réseau, probablement la lacune de cadmium.

3. Conclusion. - Nous avons présenté une technique de dérivation numérique qui procure une réjection du bruit de $18 \mathrm{~dB} /$ octave. Les coefficients de la fonction de transfert en $Z$ du filtre numérique étant calculés au préalable, on peut appliquer cette méthode en temps réel. Toutefois de meilleurs résultats sont obtenus par un traitement différé, appliqué dans le sens direct puis dans le sens rétrograde.

\section{Bibliographie}

[1] Kuo, F. F. et KaISER, J. F., System analysis by digital computer (Wiley) 1966.

Golden, R. M., KAISER, J. F., Design of interband sampled data filters B.S.T.J. 43, Part. 2 (1964) 1533-1546.

Borgner, R. E. et Constantinides, A. G., Introduction to digital filtering (Wiley) 1975.

[2] RADER, C. M. et GolD, B., Digital methods for sampled- data filters. Proceedings first allerton conference on circuit and systems theory, 1963, p. 221-236.

[3] Housin, M. et al., DLTS of electron traps and sensitizing centers in undcped CdS single crystals J. Appl. Phys. 52 (1981) 261.

Housin, M., Thèse de $3^{\mathrm{e}}$ cycle.

Housin, M., Communication interne. 